\title{
Optical Tomography for Supernovae
}

\author{
A new optical spectroscopy method can characterize the chemical composition of different \\ layers of the material ejected from type la supernovae, delivering clues to the star's history.
}

\section{by John Raymond $\dagger$}

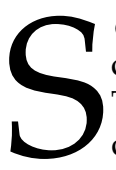

ome white dwarfs end their lives in thermonuclear explosions called type Ia supernovae (SNe). These explosions disperse atomic elements produced in the stellar cores throughout the interstellar gas-practically all elements other than hydrogen and helium are generated in the cores of stars. These elements control the gas temperature and, thereby, the formation of other stars and the evolution of galaxies. Type Ia supernovae are also used by cosmologists as standard candles for measuring astronomical distances-a method that led to the discovery of the accelerating expansion of the Universe [1]. Yet there is no scientific consensus on the stellar evolution leading to an explosion or on the explosion mechanisms themselves. Now, Ivo Seitenzahl, now at the University of New South Wales in Canberra, Australia, and colleagues report an observational technique that provides new ways to test supernova models [2]. Their method, which they dub supernova remnant tomography is based on characterizing, through optical spectroscopy, different layers of the supernova ejecta-the material ejected outward by the explosion.
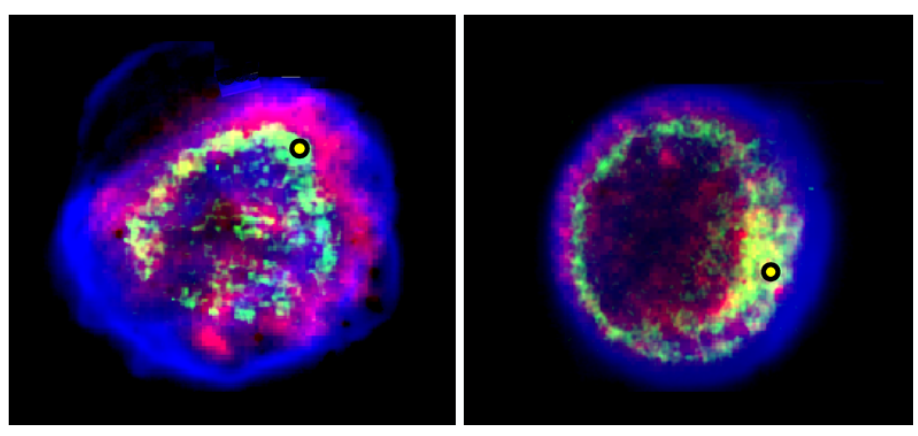

Figure 1: Optical emission images of the SNRs 0519-69.0 (left) and 0509-67.5 (right), as obtained through the "supernova remnant tomography" technique developed by Seitenzahl et al. [2]. $\mathrm{Fe}^{14+}$ emission is indicated in green, hydrogen $\alpha$ lines in blue. (I. R. Seitenzahl et al. [2])

†Center for Astrophysics | Harvard \& Smithsonian, Cambridge, MA 02138, USA
Analyzing two supernovae with their method, they find that one of them derived from a white dwarf with mass lower than the Chandrasekhar limit (1.4 solar masses)—often considered the minimum mass needed for a white dwarf to explode.

Theoretical models that describe white dwarf explosions remain full of uncertainties. For decades it was believed that masses larger than the Chandrasekhar limit were needed for the star to explode. A lighter white dwarf may be driven over the mass limit by accreting material from another white dwarf, from a red giant star, or from the core of a companion star that has been stripped of material. More recent observations and theoretical studies suggest that white dwarfs below the Chandrasekhar limit might also be able to explode, although more observations are needed to confirm this conclusion. The explosion may be initiated near the center of the star or near its surface, and the nuclear burning front may propagate subsonically or supersonically [3]. Each of these possibilities would produce different signatures in the composition or morphology of the material ejected by the star, providing a way to test models through observations.

Some stellar explosions have even been caught in the act, and large-scale surveys have now provided optical spectra of large numbers of distant supernovae. However, during the year or so during which a supernova remains bright, the expanding gas is optically thick, allowing only the composition of the outermost layers of the star to be measured. And although the makeup of the outer layer provides some information about the progenitor star, it does not directly reveal the star's bulk composition. Additional information can be derived by later-stage observations of the supernova remnant (SNR) - the ball of hot gas produced by the ejecta and by the explosion's fast-moving shock waves in the interstellar medium. The emission from the supernova remnant allows researchers to probe the ejecta composition. Such an approach is only useful for SNRs younger than a few thousand years, since for older SNRs the emission by the interstellar gas dominates over the emission by the ejecta.

To date, the analysis of SNR composition is mostly based on $\mathrm{x}$-ray spectroscopic observations that can discriminate between different elements. The gas that makes up the SNR moves at thousands of $\mathrm{km} / \mathrm{s}$, and when it encounters interstellar gas, it forms shock waves that heat the ejecta to temperatures between $10^{7}$ and $10^{9} \mathrm{~K}$. At these temperatures, 
the elements contained in the ejecta emit $\mathrm{x}$ rays, allowing researchers to analyze the ejecta's chemical composition. Such an analysis can discriminate, for instance, between type Ia SNe produced by white dwarfs and type II or type $\mathrm{Ib}$,c supernovae produced by the collapse of more massive stars. Large abundances of iron, for instance, are associated with white dwarf explosions, while the abundances of manganese and chromium can signal a particular explosion mechanism known as delayed detonation [3]. However, $x-$ ray observations are limited by the small number of $x$-ray photons available and by the spectral resolution of current instruments-often insufficient to discriminate important spectroscopic features.

The spectroscopy method reported by Seitenzahl and his co-workers provides a way to determine the composition and dynamics of ejecta from type Ia SNRs using visible, rather than $x$-ray, radiation. Their approach involves monitoring the emission from highly ionized states of iron (from $\mathrm{Fe}^{8+}$ to $\mathrm{Fe}^{13+}$ ) and of other elements. These atoms have electronic transitions at visible wavelengths, but they produce only faint emissions because the transitions are optically forbidden to first order. Despite this difficulty, researchers have managed to spot the faint lines in various environments, starting with the Sun's corona in 1942 [4].

Previous studies detected these forbidden lines in the shocked interstellar gas of a few core-collapse SNRs but not in the remnants of type Ia supernovae [5]. Seitenzahl et al. were able to take advantage of various instrumentation improvements at the Very Large Telescope (VLT) in Chile to observe these optical faint lines from type I SNRs. The improvements included the addition of a wide-field-ofview, high-spectral-resolution spectrometer (MUSE), which can acquire spectra at thousands of positions simultaneously. Observing three type Ia SNRs in the Large Magellanic Cloud-a neighboring galaxy of the Milky Way-they succeeded in measuring emission lines (Fig. 1) from several highly ionized states of iron and sulfur $\left(\mathrm{Fe}^{8+}, \mathrm{Fe}^{13+}, \mathrm{Fe}^{14+}\right.$, $\left.\mathrm{S}^{11+}\right)$. Importantly, the instrument's spectral resolution allowed them to determine the emission lines' Doppler shifts, which can be related to the ejecta's velocities at different positions. The velocity information provides a powerful test of models of the supernova explosion and the evolution of the supernova remnant.

The observations provide several tests of the theories of SN explosions and of SNR evolution. According to models for type I SNe, the progenitor star should have an onionlike structure with heavy elements at its core and lighter ones near the surface, but different explosion mechanisms create different levels of turbulent mixing between the layers. Seitenzahl et al. show sulfur emission in a layer that's outside the one dominated by iron emission in SNR 0509 (Fig. 2), but further calculations of the ionization state are required to determine the structure of the ejecta's composition. For one case (SNR 0519), the researchers find that the emis-

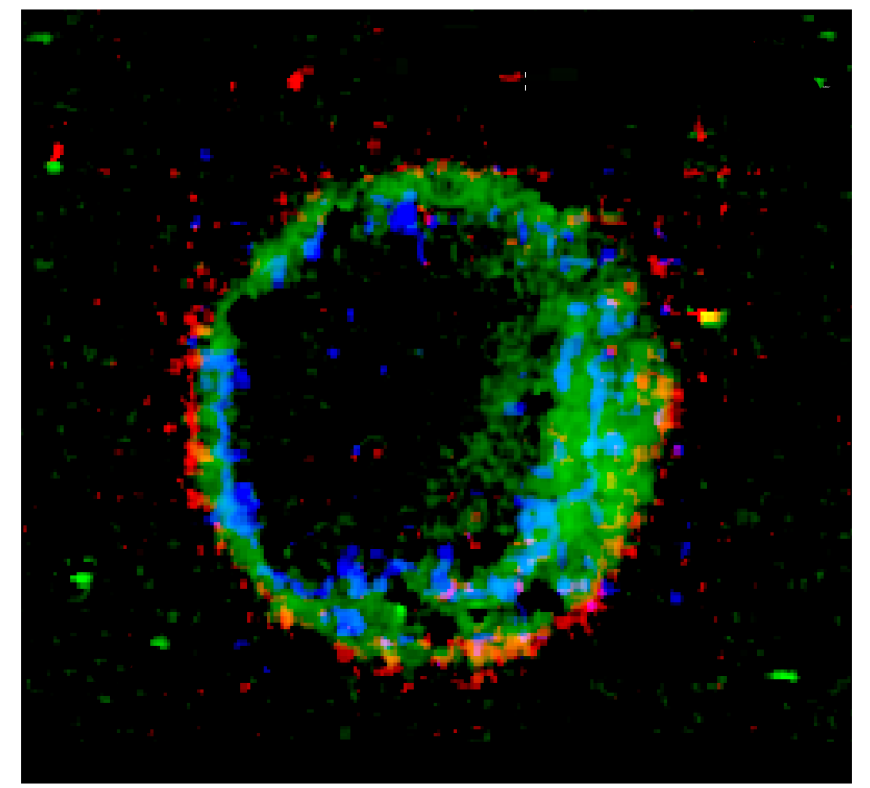

Figure 2: Image of 0509-67.5 showing emission of $\mathrm{S}^{11+}$ (red), $\mathrm{Fe}^{9+}$ (blue), and $\mathrm{Fe}^{14+}$ (green). (I. R. Seitenzahl et al. [2])

sion lines match those predicted by standard models for the explosion of a near-Chandrasekhar mass white dwarf. For SNR 0509, on the other hand, the lines indicate a progenitor with a mass below the Chandrasekhar limit. Overall, the match of the data with SNR evolution models is good, though models overpredict the Doppler broadening of the emission lines by $20 \%$ to $40 \%$.

The detection of these optical emission lines is only the first step towards fulfilling the potential of this new spectroscopy technique for $\mathrm{SNe}$ analysis. Next, theorists need to deliver model predictions for the spectral lines so that they can be compared with the observations. They will have to start with a model of the progenitor star, then describe the explosion and nuclear burning, compute the evolution of the SNR and of the associated shock wave, and, finally, derive the time-dependent abundance of the elements in the relevant ionization states. That will require a substantial effort, but it promises great rewards in terms of understanding supernova explosions and the evolution of supernova remnants.

This research is published in Physical Review Letters.

\section{REFERENCES}

[1] The Nobel Prize in Physics 2011.

[2] I. R. Seitenzahl, P. Ghavamian, J. M. Laming, and F. P. A. Vogt, "Optical tomography of chemical elements synthesized in type la supernovae," Phys. Rev. Lett. 123, 041101 (2019). 
[3] K. Nomota and S.-C. Leung, "Thermonuclear explosions of Chandrasekhar mass white dwarfs," in Handbook of Supernovae, edited by A. W. Alsabti and P. Murdin (Springer, Cham, 2017), p. 1275.

[4] B. Elden, "Der deutung de emissionslinien im spektrum der sonnenkorona," Z. Astrophysics 22, 30 (1942).
[5] M. A. Dopita, F. P. A. Vogt, R. S. Sutherland, I. R. Seitenzahl, A. J. Ruiter, and P. Ghavamian, "Shocked interstellar clouds and dust grain destruction in the LMC supernova remnant N132D," Astrophys. J. Suppl. S 237, 10 (2018).

10.1103/Physics.12.82 